\title{
Neumotórax a tensión en paciente recuperado de infección por SARS CoV-2
}

\section{Tension pneumothorax in patient recovered from infection by SARS Cov 2}

${ }^{1}$ Pedro Rafael Correa Calderón, ${ }^{2}$ Adriana Maritza León Díaz.

\section{Resumen}

Desde el inicio de la pandemia en 2020 con el aumento de casos de neumonía multilobar por enfermedad por coronavirus COVID-19, se han reportado con más frecuencia casos de neumotórax en la literatura mundial, pero a la fecha no se ha reportado ninguno de un paciente convaleciente en casa por la infección, que debute con neumotórax a tensión en urgencias.

Se trata de un paciente de 48 años, que supera la infección por COVID-19 y es dado de alta, quedando con secuelas estructurales y funcionales, la más importante, la dependencia al oxígeno a bajo flujo. Asiste al servicio de urgencias con disnea progresiva y se diagnostica neumotórax a tensión, requiriendo toracostomía cerrada, reintervención por nuevo neumotórax intrahospitalario y es dado de alta 18 días después.

En conclusión, la enfermedad por coronavirus tiene una amplia gama de presentaciones y en nuestro portafolio de diagnósticos diferenciales en el servicio de urgencias, debemos añadir esta causa de disnea en pacientes con infecciones activas y convalecientes de la enfermedad, cuyo manejo es estrictamente quirúrgico.

Palabras claves: Neumotórax a tensión; enfermedad por coronavirus; SARS CoV-2; COVID-19.

\section{Abstract}

In the 2020 pandemic with the increase in cases of multilobe pneumonia due to coronavirus disease COVID-19, cases of pneumothorax have been reported in the world literature with increasing frequency, but to date no case of a patient, convalescing at home due to the infection, who presented with tension pneumothorax in the emergency room.

This is a 48-year-old patient who overcame COVID 19 infection and was discharged, leaving with structural and functional sequelae, the most important of which was dependence on low-flow oxygen.

${ }^{1}$ Médico general. Hospital Universitario los Comuneros. Orcid: https://orcid.org/0000-00015283-0127

${ }^{2}$ Cirujana General

Hospital Universitario Los Comuneros

Autor de correspondencia Pedro Rafael Correa Calderón, correo: peracoca@hotmail.com

Recibido: $01 / 02 / 2021$

Aceptado: 09/11/2021 
He attended the emergency department with progressive dyspnea and a tension pneumothorax was diagnosed, requiring closed thoracostomy, reoperation for a new in-hospital pneumothorax, and discharged 18 days later. In conclusion coronavirus disease has a wide range of presentations, and within our portfolio of differential diagnoses in the emergency department we must add this cause of dyspnea in patients with active and convalescent infections of the disease, whose management is strictly surgical.

Keywords: Tension pneumothorax, coronavirus disease, Sars Cov 2, covid 19

\section{Introducción}

La pandemia viral del2020 porelCOVID-19 cambió paradigmas en la atención de pacientes en urgencias a lo largo del mundo y ha obligado a la ciencia a avanzar rápidamente, para intentar cubrir todos los aspectos de la enfermedad desde la prevención, hasta el tratamiento y manejo en la convalecencia. En este último aspecto se ha empezado a describir en la literatura el síndrome "post COVID" como un conjunto de secuelas, en su mayoría de naturaleza crónica, que en ocasiones motivan al paciente recuperado a asistir al servicio de urgencias, principalmente por fatiga (1).

$\mathrm{Al}$ igual, un paciente en urgencias con disnea, puede estar cursando con la complicación severa más frecuente de la infección por COVID-19, el tromboembolismo pulmonar, descrito en la literatura en el $15 \%$ de los pacientes diagnosticados con la infección (2). El neumotórax es una causa de disnea poco frecuente en pacientes con COVID-19 (35) y generalmente se ve junto a neumomediastino en pacientes ventilados mecánicamente $(5,6)$. El neumotórax a tensión es aún más infrecuente, solo con reportes de caso referenciados ocasionalmente en la literatura (7).

\section{Reporte de Caso}

Se trata de un paciente de 48 años cuyo único antecedente es litiasis renal bilateral, quien estuvo ingresado desde el 20 de octubre de 2020 por síntomas respiratorios, tos, disnea y fiebre de 10 días de evolución. Se confirmó diagnóstico de infección SARS CoV-2 por prueba de antígeno positivo que evoluciona hacia una neumonía multilobar severa (Figura 2) y requirió soporte de oxígeno por máscara de no reinhalación.

\section{$09 / 12 / 2020$}

Se sospecha neumonía intersticial no especificada (NINE) se inicia manejo con prednisona $25 \mathrm{mg}$ VO día

$05 / 12 / 2020$

- $\quad$ Ingreso a servicio de urgencias

- 5 días de disnea

- $\quad$ Agio tomografía de tórax: neumotórax a tensión

$08 / 12 / 2020$

- $\quad$ Hipoventilación izquierda

- $\quad$ Persistencia de requerimiento de $\mathrm{O} 2$ a alto flujo

- Tomografía de tórax de control: expansión completa del hemitórax neumotórax apical ipsilateral de $4 \mathrm{~mm}$

\section{$14 / 12 / 2020$}

- Desaturación y disnea súbita

- RX de tórax: neumotórax a tensión

\section{3/12/2020}

- $\quad$ Egreso a casa con O2 e indicación de completar 25 días de corticoide oral

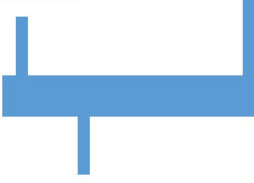

Se retira toracotomía cerrada
$22 / 12 / 2020$

- $\quad$ Se logra destete de O2 hasta cánula nasal a tres litros minuto

- $\quad$ Adecuada expansión

Figura 1. Línea del tiempo 


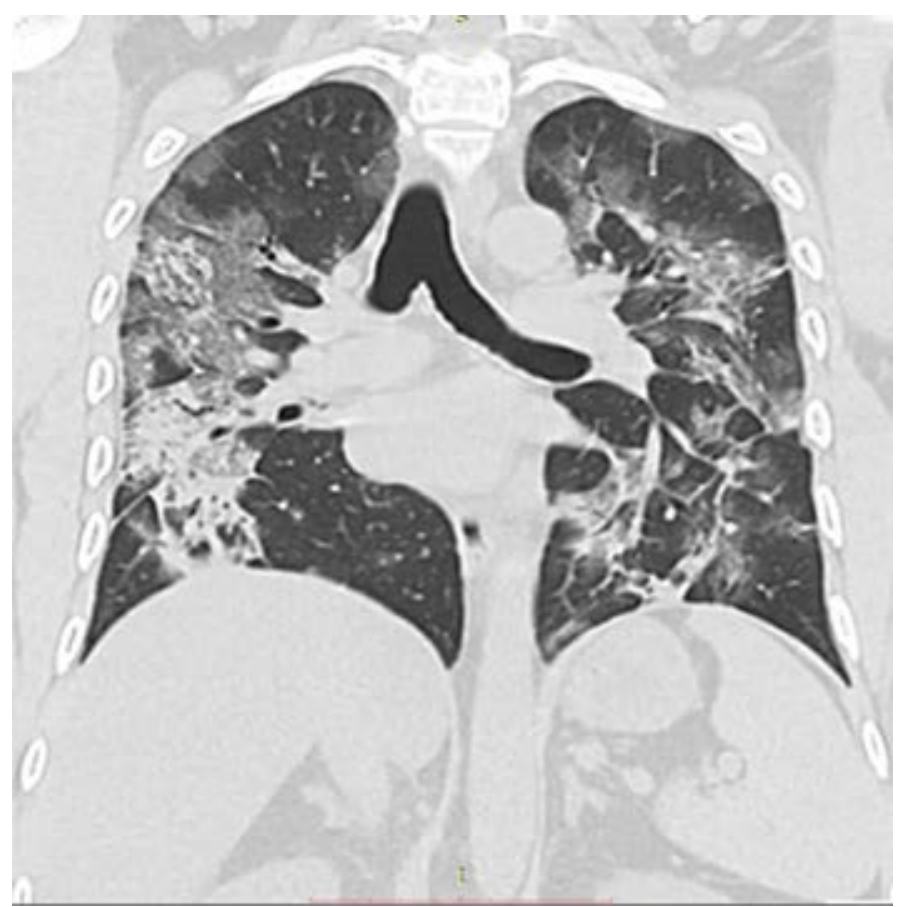

Figura 2. Prueba de antígeno positivo que evoluciona hacia una neumonía multilobar severa.

Presentó deterioro en la ventilación el 2 de noviembre de 2020 por NEWS 2 SCORE (predictor del deterioro del paciente en el ambiente hospitalario) (8) de 11 puntos e índice de ROX (predicción de fracaso de la oxigenoterapia de alto flujo por cánula nasal) (9) de 4.7. Es trasladado a la unidad de cuidado intensivo, donde permaneció hasta el 6 de noviembre de 2020; no requirió de intubación orotraqueal. Tuvo una evolución lenta hacia la mejoría, fue dado de alta el 23 de noviembre de 2020 con oxígeno domiciliario por cánula nasal, tromboprofilaxis por 10 días, prednisolona oral 15 miligramos día e inhaladores de bromuro de ipratropio y beclometasona.

El paciente consulta a urgencias el 5 de diciembre de 2020 por clínica de cinco días de evolución de disnea y aumento de requerimiento de flujo de oxigeno domiciliario, sin fiebre, tos, ni dolor torácico.

$\mathrm{Al}$ examen clínico de ingreso a urgencias el paciente presenta taquicardia, taquipnea y pulsioximetrÍa baja (signos vitales de ingreso: tensión arterial 120/90 $\mathrm{mm} \mathrm{Hg}$, frecuencia cardiaca de 148 latidos por minuto, saturación de oxigeno $75 \%$ con cánula nasal (91\% con máscara de no reinhalación), frecuencia respiratoria 36 respiraciones por minuto, temperatura $36{ }^{\circ} \mathrm{C}$. A la valoración se encontró paciente con dificultad para respirar, con uso de músculos accesorios, a la auscultación con disminución de los ruidos respiratorios en ambas bases pulmonares, sin otro hallazgo llamativo al examen físico.

En el abordaje inicial del cuadro clínico, se realiza analítica sanguínea completa (Tabla 1) y estudio con tomografía computarizada de tórax más angiografía de urgencia (Figura 3).

Inicialmente se sospechó proceso tromboembólico al pulmón por el cuadro clínico de ingreso. Dada la inestabilidad respiratoria se indicó angiotomografía de vasos pulmonares urgente, sin embargo, la imagen solicitada descartó embolismo pulmonar y evidenciaba un neumotórax a tensión izquierdo.

$\mathrm{Al}$ ingreso se realizó manejo de soporte inicial con oxígeno por medio de máscara de reservorio a 15 litros por minuto; se optimizó manejo médico con solución Hartmann 500 centímetros cúbicos en bolo e infusión a 100 centímetros cúbicos por hora, una dosis única de dexametasona 6 miligramos endovenoso, heparina de bajo peso molecular 60 miligramos subcutáneo y manejo de terapia respiratoria con inhaladores beclometasona y bromuro de Ipratropio.

Una vez se llega al diagnóstico se solicita la valoración al servicio de cirugía general el cual practica de inmediato, en sala de quirófanos, toracostomia cerrada con tubo 34 en el quinto espacio intercostal con línea axilar anterior izquierda, procedimiento llevado a cabo sin complicaciones y con la mejoría esperada en el estado clínico del paciente.

Después del procedimiento se realiza manejo en conjunto intrahospitalario entre medicina interna y cirugía general. Se brinda manejo médico con analgésicos, tromboprofilaxis y terapia respiratoria, se corrobora al siguiente día adecuada expansión del hemitórax izquierdo (Figura 4). Llama la atención que a pesar de esto el paciente persiste con requerimiento de oxígeno a alto flujo, por lo cual al tercer día postoperatorio se realiza tomografía de tórax de control (Figura 5) en la cual solo se describe neumotórax apical izquierdo de 4 milímetros. Al quinto día es valorado por el servicio de neumología el cual considera que el paciente cursa con neumonía intersticial no especificada y se beneficia del inicio de prednisona 25 miligramos por vía oral, diariamente por un mes. Finalmente al sexto día de manejo intrahospitalario, ante la mejoría clínica y la disminución del flujo de oxígeno, se retira tubo de tórax, continuando bajo vigilancia intrahospitalaria. 
Tabla 1. Analítica sanguínea.

\begin{tabular}{|ll|}
\hline \multicolumn{1}{|c|}{ Nombre del Estudio } & \multicolumn{1}{c|}{ Resultado } \\
Creatinina & $0.66 \mathrm{mg} / \mathrm{dl}$ \\
BUN & $15.8 \mathrm{mg} / \mathrm{dl}$ \\
Ácido Láctico (gases & $3.2 \mathrm{mmol} / \mathrm{L}$ \\
arteriales) & \\
PaO2 (gases arteriales) & $54.5 \mathrm{mmHg}$ \\
PCO2 (gases arteriales) & $34 \mathrm{~mm} \mathrm{Hg}$ \\
HCO3 (gases arteriales) & $21.4 \mathrm{mmol} / \mathrm{L}$ \\
PH (gases arteriales) & 7.412 \\
Cloro & $103 \mathrm{mmol} / \mathrm{L}$ \\
Sodio & $140 \mathrm{mmol} / \mathrm{L}$ \\
Potasio & $4.48 \mathrm{mmol} / \mathrm{L}$ \\
Dímero D & $1.37 \mathrm{mg} / \mathrm{L}$ \\
INR & 0.92 \\
PTT & $27.3 \mathrm{~s}$ \\
Glóbulos Blancos & $10790 / \mathrm{microlitro}$ \\
Neutrófilos & $71.6 \%$ \\
Hemoglobina & $13.76 \mathrm{~g} / \mathrm{dl}$ \\
Plaquetas & $283000 / \mathrm{microlitro}$ \\
\hline
\end{tabular}

Tres días más tarde el paciente vuelve a presentar desaturación y disnea súbita; se practica radiografía de tórax donde se aprecia un nuevo neumotórax a tensión izquierdo (Figura 6). Nuevamente se pasa a quirófano a toracostomía cerrada izquierda, se optimizó la terapia respiratoria por una semana más y finalmente se retiró el tubo el 22 de diciembre de 2020.

Se da de alta al día siguiente con indicación de completar el mes de esquema de corticoide oral y oxigeno por cánula nasal a bajo flujo. Se dejó control con neumología en dos meses con una nueva tomografía.

\section{Discusión}

El caso se afronta de ingreso erróneamente como un tromboembolismo pulmonar; no se considera la posibilidad del neumotórax a tensión, dada su baja prevalencia y es por esto que no se realiza el manejo indicado en estos casos, el cual es drenaje de aire con catéter 12-14 en el segundo espacio intercostal, para estabilizar el paciente y posterior paso a toracostomía cerrada. En el mejor de los casos no se necesita imagen y si eventualmente esta llegara a ser necesaria, no debería ser una tomografía, sino una radiografía de tórax simple (10).

Sin embargo, afortunadamente se contó con oportunidad en el diagnóstico y manejo, logrando finalmente que el paciente pudiera ser egresado a casa sin secuelas adicionales a las dejadas por la infección inicial.

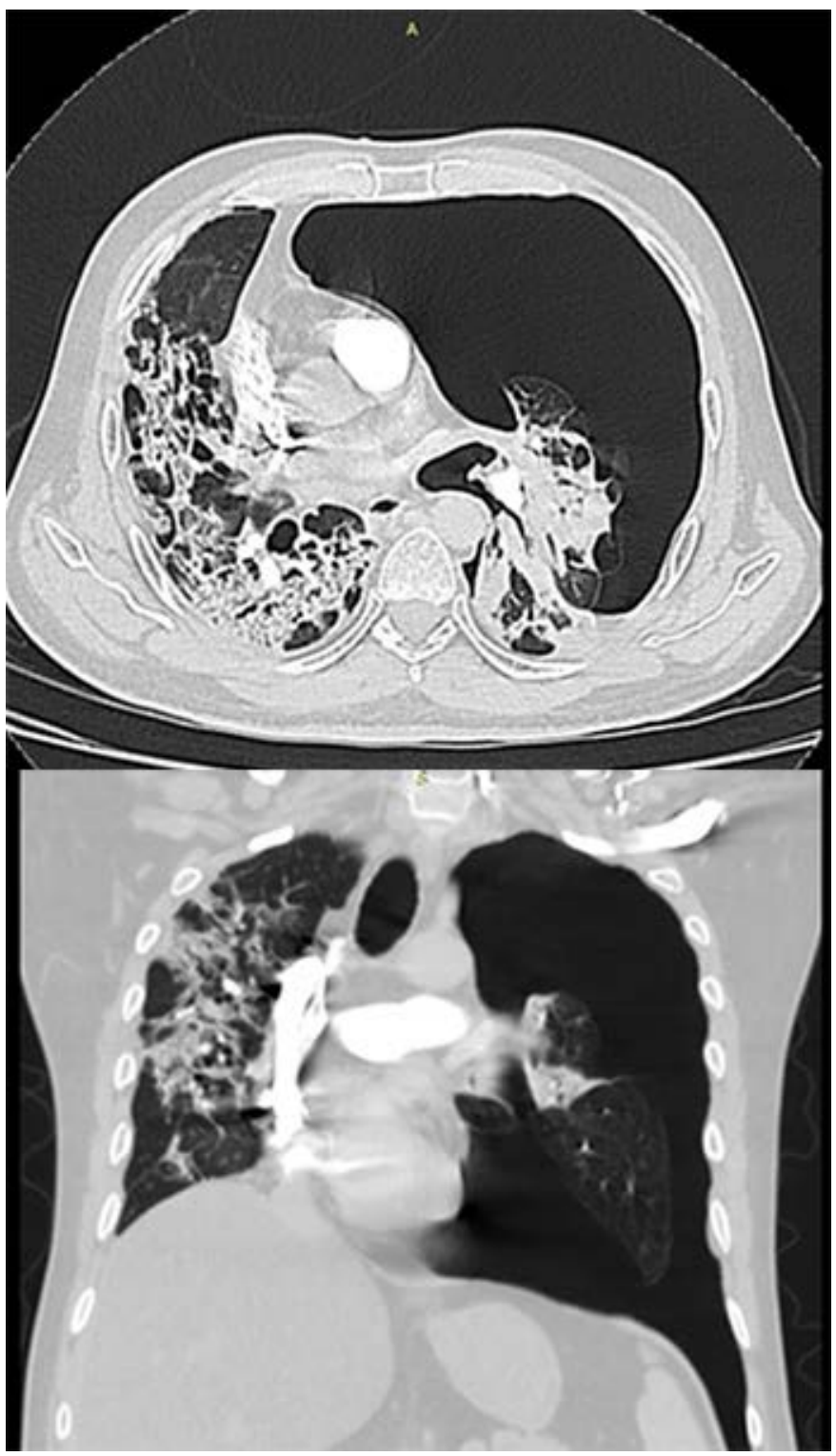

Figura 3. Tomografía computarizada de tórax más angiografía de urgencia. 


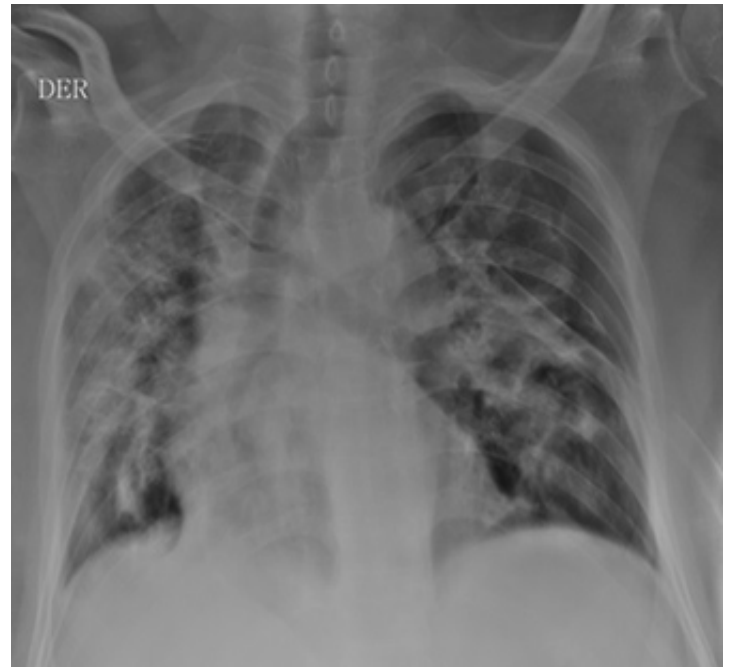

Figura 4. Expansión del hemitórax izquierdo.
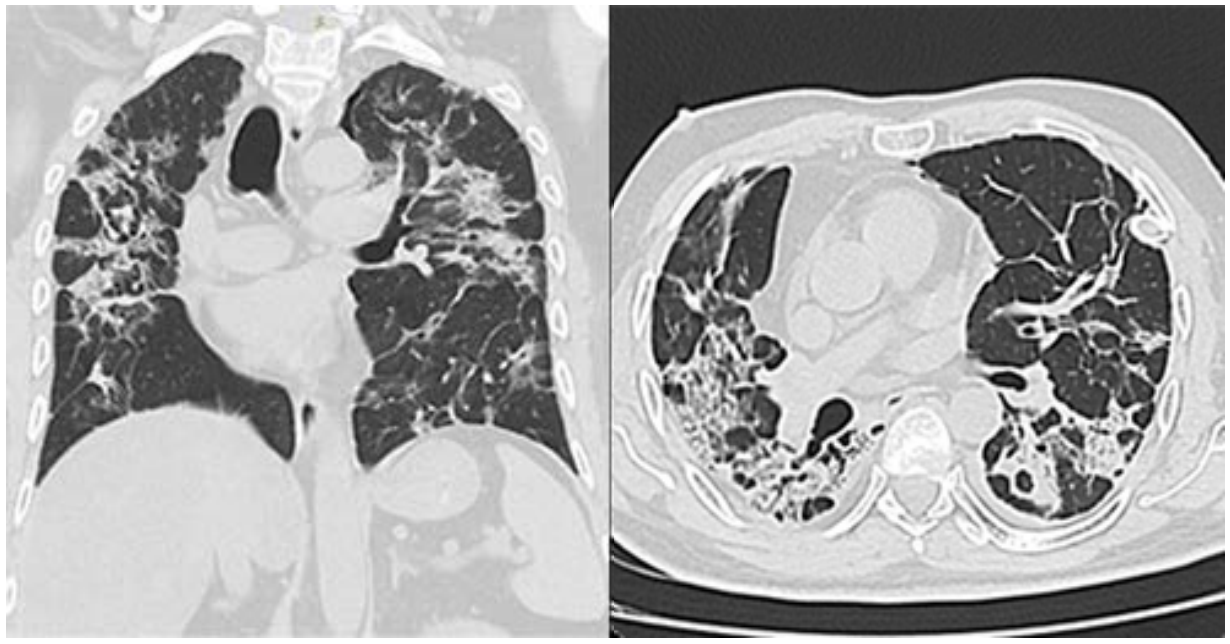

Figura 5. Tomografía de tórax de control.

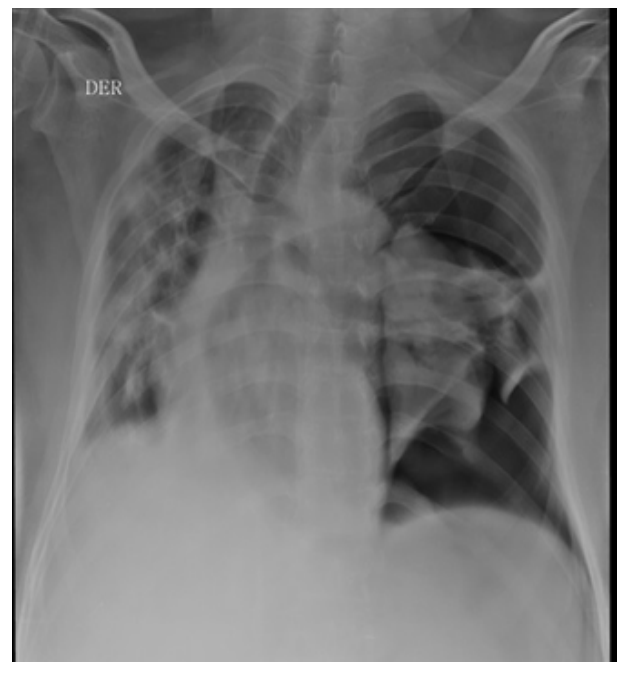

Figura 6. Radiografía de tórax donde se aprecia el nuevo neumotórax a tensión izquierdo. 
Es muy importante para médicos generales, internistas, urgenciólogos y cirujanos, conocer de esta condición en el contexto de la pandemia por COVID-19, ya que de otra manera, si el paciente con neumotórax a tensión se deteriora y se decide abordar la vía aérea por medio de intubación orotraqueal, muy probablemente se hubiera generado un paro cardiorrespiratorio iatrogénico empeorando el desenlace final del paciente.

La infección por COVID-19 nos ha enseñado una amplia gama de presentaciones, desde las más leves hasta las más severas y ha involucrado las diferentes especialidades de la medicina en esta batalla por la vida. Se considera que este paciente generó una fibrosis pulmonar tan severa, que a pesar de no haber requerido ventilación mecánica, desarrolló una fuga de aire al espacio pleural que por efecto válvula e insidiosamente se convirtió en un neumotórax a tensión, requiriendo manejo quirúrgico con toracostomía cerrada en dos ocasiones. Necesitamos tiempo y cohortes para poder caracterizar las secuelas pulmonares a mediano y largo plazo de esta nueva enfermedad; de momento, día a día continuamos indagando en la literatura con novedades en pro de nuestro buen actuar y el beneficio del paciente.

\section{Referencias}

1. Kamal M, Abo Omirah M, Hussein A, Saeed H. Assessment and characterisation of post-COVID-19 manifestations. Int J Clin Pract. 2021 Mar;75(3):e13746. doi: 10.1111/ijcp.13746.

2. Liao SC, Shao SC, Chen YT, Chen YC, Hung MJ. Incidence and mortality of pulmonary embolism in COVID-19: a systematic review and meta-analysis. Crit Care. 2020 Jul 27;24(1):464. doi: 10.1186/s13054-020-03175-z.

3. Chen N, Zhou M, Dong X, Qu J, Gong F, Han Y, et al. Epidemiological and clinical characteristics of 99 cases of 2019 novel coronavirus pneumonia in Wuhan, China: a descriptive study. Lancet. 2020 Feb 15;395(10223):507-513. doi: 10.1016/ S0140-6736(20)30211-7.

4. Chang T, Wu J, Chang L. Clinical Course and outcomes of critically ill patients with COVID19 in Wuhan China. J Formos Med Assoc. 2020;(January):19-21.

5. Zantah M, Dominguez Castillo E, Townsend R, Dikengil F, Criner GJ. Pneumothorax in COVID-19 disease- incidence and clinical characteristics. Respir Res. Respiratory Research; 2020;21(1):1-9.

6. Martinelli AW, Ingle $T$, Newman J, Nadeem I, Jackson K, Lane ND, et al. COVID-19 and pneumothorax: a multicentre retrospective case series. Eur Respir J. 2020;56(5). http://dx.doi. org/10.1183/13993003.02697-2020

7. Flower L, Carter JPL, Rosales Lopez J, Henry AM. Tension pneumothorax in a patient with COVID-19. BMJ Case Rep. 2020;13(5):1-4.

8. Indd N. (2017). National Early Warning Score (NEWS) 2 Standardising the assessment of acute-illness severity in the NHS. www.rcplondon. ac.uk

9. Roca O, Caralt B, Messika J, Samper M, Sztrymf B, Hernández G, García-De-Acilu M, Frat JP, Masclans JR, Ricard JD. An index combining respiratory rate and oxygenation to predict outcome of nasal high-flow therapy. American Journal of Respiratory and Critical Care Medicine. 2019;199(11):1368-1376. https://doi. org/10.1164/rccm.201803-0589OC

10. Bauman MH, Strange C, Heffner JE, Light R, Kirby TJ, Klein J, et al. Management of spontaneous pneumothorax: An American College of Chest Physicians Delphi Consensus Statement. Chest. 2001;119(2):590-602. 